\title{
AN APPLICATION OF HADAMARD-LÉVY'S THEOREM TO A SCALAR INITIAL VALUE PROBLEM
}

\author{
SORIN RĂDULESCU AND MARIUS RĂDULESCU
}

(Communicated by Kenneth R. Meyer)

\begin{abstract}
A global inversion theorem due to Hadamard and Lévy is applied to a Volterra integral operator acting in a space of continuous functions to obtain a one-sided criterion for global existence and uniqueness of a scalar initial value problem.
\end{abstract}

The aim of the present paper is to establish an existence and uniqueness theorem for a scalar initial value problem, by using a global inversion theorem due to Hadamard and Lévy.

We obtain a one-sided condition which improves a special case of a previous result established in [8].

The following theorem is known as the Hadamard-Lévy theorem.

Theorem 1. Let $E, F$ be two Banach spaces and $f: E \rightarrow F$ be a $C^{1}$ map for which

(1) $f^{\prime}(x) \in \operatorname{Isom}(E, F)$ for every $x \in E$. If there exists a continuous map $\omega: \mathbf{R}_{+} \rightarrow \mathbf{R}_{+}^{*}$ such that

(2) $\int_{0}^{\infty} \frac{d s}{\omega(s)}=+\infty$,

(3) $\left\|\left[f^{\prime}(x)\right]^{-1}\right\| \leq \omega(\|x\|), x \in E$,

then $f$ is a $C^{1}$ global diffeomorphism. Here by a $C^{1}$ global diffeomorphism we understand a one-to-one map which is $C^{1}$, onto and whose inverse is also a $C^{1}$ map. By $\operatorname{Isom}(E, F)$ we denote the set of all linear continuous isomorphisms of $E$ onto $F$.

One can easily see that for some positive constants $a, b$ the maps $\omega_{1}(t)=1$, $\omega_{2}(t)=a t+b, \omega_{3}(t)=(a t+b) \ln (t+2), \omega_{4}(t)=(a t+b) \ln (t+2) \ln \ln (t+3)$, $t \geq 0$ satisfy condition (2).

A proof of Theorem 1 may be found in $[2,6-9,11]$. Here we have presented a somewhat more general statement cf. [5]. In [1, 3, 4, 8-11], the Hadamard-Lévy

Received by the editors July 26, 1987, and, in revised form, July 20, 1988.

1980 Mathematics Subject Classification (1985 Revision). Primary 34G20; Secondary 58C15.

Key words and phrases. Global inversion theorem, $C^{1}$ global diffeomorphism, scalar initial value problem, global solution. 
theorem was used to obtain existence and uniqueness theorems for nonlinear boundary value problems.

Throughout the paper $J$ will denote the closed bounded interval $[a, b]$ and $F$ will denote the Banach space of all real continuous functions defined on $J$, endowed with the usual sup-norm.

Lemma 2. Let $v \in F, \quad v_{1}=\frac{1}{2}(v+|v|)=\max (v, 0)$ and $c=$ $\exp \left(\sup _{t \in J} \int_{a}^{t} v(s) d s\right)$. Consider the linear bounded operator $T: F \rightarrow F$ defined as

$$
(T k)(t)=k(t)+\int_{a}^{t} \exp \left(\int_{s}^{t} v(\tau) d \tau\right) v(s) k(s) d s \quad k \in F, t \in J .
$$

Then the following assertions hold

(i) $c \leq\|T\| \leq 2 \exp \left(\int_{a}^{b} v_{1}(s) d s\right)$;

(ii) $\|T\|=c$ provided that $v \geq 0$;

(iii) the map $h=T k$ is the unique solution in $F$ of the integral equation

$$
h(t)-\int_{a}^{t} v(s) h(s) d s=k(t) \quad t \in J .
$$

Proof. Denote $w(s, t)=\exp \left(\int_{s}^{t} v(\tau) d \tau\right), w_{1}(s, t)=\exp \left(\int_{s}^{t} v_{1}(\tau) d \tau\right), s, t \in J$. One can easily see that

$$
\begin{gathered}
\int_{a}^{t} w(s, t) v(s) d s=w(a, t)-1 \quad t \in J \\
\int_{a}^{t} w_{1}(s, t) v_{1}(s) d s=w_{1}(a, t)-1 \quad t \in J \\
w(s, t) \leq w_{1}(s, t) \quad a \leq s \leq t \leq b .
\end{gathered}
$$

Let $t \in J$ and $k \in F$. Then by (6), (7) and (8) we obtain

$$
\begin{aligned}
\mid(T k) & (t) \mid \leq\|k\|\left(1+\int_{a}^{t} w(s, t)|v(s)| d s\right) \\
= & \|k\|\left(1+\int_{a}^{t} w(s, t)\left(2 v_{1}(s)-v(s)\right) d s\right) \\
& =\|k\|\left(1+2 \int_{a}^{t} w(s, t) v_{1}(s) d s-\int_{a}^{t} w(s, t) v(s) d s\right) \\
& \leq\|k\|\left(2-w(a, t)+2 \int_{a}^{t} w_{1}(s, t) v_{1}(s) d s\right) \\
& =\|k\|\left(2 w_{1}(a, t)-w(a, t)\right) \leq 2\|k\| w_{1}(a, b) .
\end{aligned}
$$

Thus the right inequality in assertion (i) is proved. Let $k_{0} \in \mathbf{R}$ and $k(t)=k_{0}$ for every $t \in J$. Then

$$
(T k)(t)=k_{0}\left(1+\int_{a}^{t} w(s, t) v(s) d s\right)=k_{0} w(a, t) \quad t \in J
$$


whence $\|T k\|=c\|k\|$. Thus the proof of the left inequality in (i) is complete. Assertion (ii) follows at once from (i) and from the inequality

$$
|(T k)(t)| \leq\|k\|\left(1+\int_{a}^{t} w(s, t) v(s) d s\right) \quad t \in J, k \in F .
$$

By Banach's contraction principle (cf. [8, Lemma 3.1]), equation (5) has a unique solution. Integration by parts shows that the map $h=T k$ verifies (5).

It would be interesting to know whether assertion (ii) holds without the assumption $v \geq 0$.

Theorem 3. Let $f: J \times \mathbf{R} \rightarrow \mathbf{R}$ be a continuous map for which its partial derivative with respect to the second argument, denoted by $f_{x}^{\prime}$, exists and is continuous. Suppose that there exist an integrable map $g: J \rightarrow \mathbf{R}_{+}$and a continuous increasing map $\omega: \mathbf{R}_{+} \rightarrow(1, \infty)$ for which the following conditions hold

$$
\begin{gathered}
\int_{0}^{\infty} \frac{d s}{\omega(s)}=+\infty \\
f_{x}^{\prime}(t, x) \leq g(t) \ln \omega(|x|) \quad t \in J, x \in \mathbf{R} .
\end{gathered}
$$

The for every $x_{0} \in \mathbf{R}$ the initial value problem

$$
\dot{x}=f(t, x), \quad x(a)=x_{0}
$$

has a unique global solution $x: J \rightarrow \mathbf{R}$.

Proof. Let $x_{0} \in \mathbf{R}$ and define $V: F \rightarrow F$ as

$$
(V x)(t)=x(t)-x_{0}-\int_{a}^{t} f(s, x(s)) d s, \quad t \in J, x \in F .
$$

Note that $V$ is of $C^{1}$ class,

$$
V^{\prime}(x)(h)(t)=h(t)-\int_{a}^{t} f_{x}^{\prime}(s, x(s)) h(s) d s, \quad t \in J, x, h \in F
$$

and $V^{\prime}(x) \in \operatorname{Isom}(F, F)$ for every $x \in F$.

Consider the equation $V^{\prime}(x)(h)=k$ where $x, k \in F$ are supposed to be known. Then by Lemma 2 the unique solution of the above equation is

$$
h(t)=k(t)+\int_{a}^{t} \exp \left(\int_{s}^{t} f_{x}^{\prime}(\tau, x(\tau)) d \tau\right) \cdot f_{x}^{\prime}(s, x(s)) k(s) d s, \quad t \in J,
$$

and

$$
\left\|\left[V^{\prime}(x)\right]^{-1}(k)\right\|=\|h\| \leq 2 \exp \left(\int_{a}^{b} \max \left(f_{x}^{\prime}(s, x(s)), 0\right) d s\right) .
$$

By (10) we obtain

$$
\left\|\left[V^{\prime}(x)\right]^{-1}\right\| \leq 2 \exp \left(\int_{a}^{b} g(s) \ln \omega(\|x\|) d s\right) \quad x \in F
$$


If $\int_{a}^{b} g(s) d s \leq 1$, then it follows from (11) that

$$
\left\|\left[V^{\prime}(x)\right]^{-1}\right\| \leq 2 \omega(\|x\|), \quad x \in F .
$$

Then Hadamard-Lévy theorem implies that $V$ is a $C^{1}$ global diffeomorphism, whence the equation $V x=0$ has a unique solution $x \in F$, which is the unique solution of (11).

Next, consider the case $\int_{a}^{b} g(s) d s>1$. Let $a=t_{0}<t_{1}<t_{2}<\cdots<t_{n}=b$ be a division of $J$ such that

$$
\int_{t_{i}}^{t_{i+1}} g(s) d s \leq 1, \quad i=0,1, \ldots, n-1 .
$$

Note that

$$
f_{x}^{\prime}(t, x) \leq g(t) \ln \omega(|x|), \quad t \in\left[t_{i}, t_{i+1}\right], x \in \mathbf{R}, \quad . \quad i \in\{0,1, \ldots, n-1\} .
$$

By (13) and (14) there exists a unique solution $\varphi_{0}:\left[t_{0}, t_{1}\right] \rightarrow \mathbf{R}$ for the initial value problem

$$
\dot{x}=f(t, x), \quad x\left(t_{0}\right)=x_{0} .
$$

Denote $x_{1}=\varphi_{0}\left(t_{1}\right)$. By (13) and (14) there exists a unique solution $\varphi_{1}$ : $\left[t_{1}, t_{2}\right] \rightarrow \mathbf{R}$ for the intial value problem

$$
\dot{x}=f(t, x), \quad x\left(t_{1}\right)=x_{1} .
$$

Construct inductively the solutions $\varphi_{i}:\left[t_{i}, t_{i+1}\right] \rightarrow \mathbf{R}, i=1,2, \ldots, n-1$, of the initial value problems

$$
\dot{x}=f(t, x), \quad x\left(t_{i}\right)=x_{i},
$$

where $x_{i}=\varphi_{i-1}\left(t_{i}\right)$.

One can easily see that the map $\varphi(t)=\varphi_{i}(t), t \in\left[t_{i}, t_{i+1}\right], i \in\{0,1, \ldots$, $n-1\}$ is of $C^{1}$ class, verifies the equations $\dot{\varphi}(t)=f(t, \varphi(t)), t \in J, \varphi(a)=x_{0}$, and is the unique map possessing these properties.

The above theorem improves a special case (namely $E=\mathbf{R}$ ) of the following theorem:

Theroem 4[8, Theorem 3.3]. Let $E$ be a Banach space and $f:[0, a] \times E \rightarrow$ $E$ a continuous map for which its partial derivative with respect to the second argument, denoted by $f_{x}^{\prime}$, exists and is continuous.

Suppose that there exist a constant $k>0$ and a continuous increasing map $\omega: \mathbf{R}_{+} \rightarrow[1, \infty)$ satisfying condition (9) and a constant $k>0$ such that

$$
\left\|f_{x}^{\prime}(t, x)\right\| \leq k \ln \omega(\|x\|), \quad t \in[0, a], x \in E .
$$

Then for every $x_{0} \in E$ the initial value problem

$$
\dot{x}=f(t, x), \quad x(0)=x_{0}
$$


has a unique global solution $x:[0, a] \rightarrow E$.

Remarks. One can easily see that there is a class of functions $f: J \times \mathbf{R} \rightarrow \mathbf{R}$ for which the growth condition (10) on $f_{x}^{\prime}$ holds but the Wintner condition [12]:

$$
|f(t, x)| \leq g(t) \omega(|x|), \quad t \in J, x \in \mathbf{R}
$$

where $g$ is integrable and $\omega$ verifies (9), does not hold. See for example rapidly decreasing maps such as $f_{1}(t, x)=-e^{x}$ or $f_{2}(t, x)=-x^{n}|x|, t \in J, x \in \mathbf{R}$ where $n$ is a positive odd number.

Note that there are also functions $f: J \times \mathbf{R} \rightarrow \mathbf{R}$ such as $f(t, x)=\sin x^{2}$, $t \in J, x \in \mathbf{R}$ for which Wintner condition (14) holds but which does not verify the growth condition (10) with $\omega$ subject to (9).

\section{REFERENCES}

1. V. Alexiades, A. R. Elcrat and P. W. Schaefer, Existence theorems for some nonlinear fourth order elliptic boundary value problems, Nonlinear Anal. 4 (1980), 805-813.

2. M. S. Berger, Non-linearity and functional analysis, Academic Press, New York, 1977.

3. K. J. Brown, Nonlinear boundary value problems and a global inverse function theorem. Ann. Math. Pura Appl. 106 (1975), 205-214.

4. K. J. Brown and S. S. Linn, Periodically perturbed conservative systems and a global inverse function theorem, Nonlinear Anal. 4 (1980), 193-201.

5. M. Cristea, A note on global inversion theorems and applications to differential equations, Nonlinear Anal. 5 (1981), 1155-1161.

6. T. Parthasarathy, On global univalence theorems. Lecture Notes in Math., vol. 977, SpringerVerlag, Berlin, New-York and Tokyo, 1983.

7. R. Plastock, Homeomorphisms between Banach spaces, Trans. Amer. Math. Soc. 200 (1974), 169-183.

8. M. Rădulescu and S. Rădulescu, Global inversion theorems and applications to differential equations, Nonlinear Anal. 4 (1980), 951-965.

9. __ Theorems and problems in analysis, Ed. Didactică şi Pedagogică, Bucureşti 1982. (Romanian)

10. A. Tineo, Global inversion theorems and applications to the existence of $2 \pi$-periodic solutions of the equation $x^{(m)}+F\left(x, \dot{x}, \ldots, x^{(m-1)}\right)=p(t)=p(t+2 \pi)$, Notas Mat. Univ. de Los Andes, Merida (Venezuela), 1978. (Preprint, Spanish)

11. __ Global inversion, Notas de Mat. Univ. de Los Andes, Merida (Venezuela), 1980. (Preprint, Spanish)

12. A. Wintner, The infinites in the non-local existence problem for ordinary differential equations, Amer. J. Math. 68 (1946), 173-178.

INSTITUTE OF MATHEMATICS, STR.ACADEMIEI 14, 70109 BUCHAREST, RoMANiA

Centre of Mathematical Statistics, Bd. Magheru 22, 70158, Bucharest, Romania 